\title{
Uso de indicador de qualidade para fundamentar programa de capacitação de profissionais de enfermagem*
}

\author{
USE OF INDICATOR OF NURSING ASSISTANCE TO BASE QUALIFICATION PROGRAM \\ USO DE INDICADOR DE CALIDAD PARA FUNDAMENTAR EL PROGRAMA DE \\ CAPACITACIÓN DE ENFERMERÍA
}

Lilian Mara Rondello Nepomuceno ${ }^{1}$, Paulina Kurcgant ${ }^{2}$

\section{RESUMO}

Trata-se de um estudo descritivo-exploratório, com o objetivo de propor um programa de capacitação para o pessoal de enfermagem, tendo por base um indicador de qualidade da assistência de enfermagem relacionado à manutenção da integridade da pele do $\mathrm{RN}$, durante um determinado período de internação hospitalar. Foram analisados os dados referentes a $121 \mathrm{RN}$ durante o período de internação na Unidade Neonatal de um hospital universitário, segundo as lesões de pele adquiridas, fatores de risco associados e registro das lesões em impressos do sistema de assistência de enfermagem. Foram identificadas 230 lesões de pele, sendo os tipos mais freqüentes equimose, eritema perineal, monilíase e, em menor número, infiltração, hematoma, erosão, fissura, escoriação, abcesso e impetigo. Os resultados fundamentaram a elaboração de um programa de capacitação apoiado nos princípios do planejamento coletivo e no desenvolvimento de competências técnico-científicas, ético-políticas e sócio-educativas. O desenvolvimento deste estudo evidenciou a importância da aplicação de indicadores de qualidade como uma das ferramentas para a avaliação do gerenciamento da assistência dos serviços prestados.

\section{DESCRITORES}

Cuidados de enfermagem.

Capacitação em serviço.

Indicadores de qualidade em assistência à saúde.

Enfermagem neonatal.

\begin{abstract}
Descriptive-exploratory study, aiming to propose a program of qualification for the nursing staff, based on an indicator of healthcare quality related to the newborn's skin integrity during a given period of hospital internment. Data from 121 newborns, collected during their stays at a neonatal unit of a university hospital according to the acquired skin injuries, associated risk factors and injury records in files of the nursing assistance system. 230 skin injuries were identified, with the most frequent types being ecchymosis, perineal erythemas, moniliasis, and, in lower amounts, infiltrations, bruises, erosion, fissures, excoriations, abscesses and impetigo. The results served as the base for the elaboration of a qualification program supported on the principles of the collective planning and development of technical-scientific, ethicalpolitical and socio-educational competences. The development of this study made it evident that it is important to apply indicators of quality as one of the tools to evaluate the management of healthcare provision.
\end{abstract}

\section{KEY WORDS}

Nursing care.

Inservice training.

Quality indicators, health care.

Neonatal nursing.

\section{RESUMEN}

Se trata de un estudio descriptivo-exploratorio, con el objetivo de proponer un programa de capacitación sobre la asistencia para el personal de enfermería, teniendo como indicador de calidad el mantenimiento de la integridad de la piel del RN hospitalizado en un determinado periodo. Fueron analizados los datos de $121 \mathrm{RN}$ durante su internación en la Unidad Neonatal de un hospital universitario, según lesiones de piel adquiridas, factores de riesgo asociados y registro impreso de las lesiones en el sistema de asistencia de enfermería. Fueron identificados 230 lesiones de piel, siendo los tipos más frecuentes equimosis, eritema perineal, moniliasis y en menor número, infiltración, hematoma, erosión, fisura, escoriación, absceso e impétigo. Los resultados fundamentaron la elaboración de un programa de capacitación apoyado en los principios de planificación colectiva y en el desarrollo de competencias técnico-científicas, ético-políticas y socio-educativas. El desarrollo de este estudio evidenció la importancia de aplicar indicadores de calidad como una herramienta para evaluar el gerenciamiento de la asistencia de los servicios ofrecidos.

\section{DESCRIPTORES}

Atención de enfermería.

Capacitación en servicio.

Indicadores de la calidad de la atención de salud.

Enfermería neonatal.

* Extraído da dissertação “Avaliação do indicador de qualidade 'integridade da pele do recém-nascido' como subsídio para a capacitação do pessoal de enfermagem", Escola de Enfermagem, Universidade de São Paulo, 2007. ${ }^{1}$ Enfermeira da Unidade Neonatal do Hospital Universitário da Universidade de São Paulo. Mestranda pelo Departamento de Administração em Enfermagem da Escola de Enfermagem, Universidade de São Paulo (EEUSP). São Paulo, SP Brasil. lirondello@yahoo.com.br ${ }^{2}$ Professora Titular do Departamento de Administração em Enfermagem da Escola de Enfermagem, Universidade de São Paulo (EEUSP). São Paulo, SP, Brasil. pkurcg@usp.br 


\section{INTRODUÇÃO}

A preocupação com a qualidade tem estado presente na história da humanidade e o seu conceito evoluiu historicamente, associado ao progresso político, econômico e cultural de cada época ${ }^{(1)}$. Na perspectiva do pensamento moderno da qualidade, esse conceito, passou a ser compreendido como parte da função gerencial e como elemento essencial para a sobrevivência das organizações nos mercados atuais que são altamente competitivos.

Da mesma forma, o desenvolvimento do conceito de qualidade aplicado aos serviços de saúde, passou por um processo evolutivo semelhante, incorporando novos elementos com o movimento histórico da sociedade e das organizações.

Assim, o conceito de garantia de qualidade em saúde refere-se à elaboração de estratégias tanto para a avaliação da qualidade quanto para a implementação de normas e padrões de conduta clínica através de programas locais ou nacionais ${ }^{(2)}$.

Para um estudioso do tema, o conceito de qualidade é atribuído à obtenção de maiores benefícios e redução dos riscos para o paciente e fundamenta a avaliação dos serviços de saúde em três grandes componentes relacionados à estrutura do serviço, aos processos e aos resultados da assistência à saúde prestada a população ${ }^{(3)}$.

Nesse sentido, esses conceitos subsidiaram a elaboração de instrumentos que visam avaliar a qualidade dos serviços prestados sendo aplicáveis também à gestão da qualidade nessa área. sistemas de informação sendo utilizados para melhorar as atividades realizadas ou mensurar o grau de risco de um evento ou agravo à saúde; para atribuir valor a dados ou aspectos da realidade que se deseja conhecer e, a partir destes conhecimentos, intervir para alcançar objetivos ${ }^{(7)}$.

A utilização de indicadores nos cenários de assistência a saúde facilita o enfrentamento do futuro, além de possibilitar a comparabilidade entre os serviços no âmbito intra e extra-hospitalar ${ }^{(5)}$.

Assim, no setor da saúde, a política da qualidade tem gerado uma preocupação constante com a melhoria da assistência prestada ao paciente, exigindo maiores investimentos na qualificação dos trabalhadores.

Essa qualificação deve estar voltada para o desenvolvimento do ser humano de forma integral e para o atendimento das necessidades identificadas em uma realidade de trabalho específica.

Desenvolver pessoas e melhorar a qualidade da assistência à saúde, beneficia tanto os trabalhadores como os pacientes, considerando seus direitos como cidadãos contribuindo para melhorar a qua-
O desenvolvimento do conceito de qualidade aplicado aos serviços de saúde passou por um processo evolutivo, incorporando novos elementos com o movimento histórico da sociedade e das organizações. lidade de vida das pessoas.

No que se refere à saúde da mulher e do recém-nascido (RN), os elevados índices de mortalidade materna e neonatal e a escassez de programas e ações de saúde são fatores desencadeantes de preocupação para os profissionais cientes de sua responsabilidade quanto à importância de prestar uma assistência de qualidade. Dessa maneira, verificase a necessidade de profissionais de enfermagem que desenvolvam ações de saúde com conhecimento, habilidade, e competência ${ }^{(8)}$.
Os instrumentos que avaliam a qualidade dos serviços de saúde e de enfermagem podem ser divididos em internos como: Comissões de Auditoria Médica e de Enfermagem, Comissão de Prevenção e Controle das Infecções Hospitalares, Comissão de Ética em Pesquisa, Comissão de Educação Continuada, Comissão de Gerenciamento de Recursos Materiais, Comissão de Gerenciamento de Risco e Comissão de Avaliação dos Usuários; e externo como a Acreditação Hospitalar ${ }^{(4)}$.

Dentre esses instrumentos, os indicadores de qualidade são imprescindíveis na avaliação de processos de trabalho, tanto no planejamento como na organização, coordenação/ direção e na avaliação das atividades desenvolvidas ${ }^{(5)}$.

O indicador é uma variável, característica ou atributo de estrutura, processo ou resultado que é capaz de sintetizar, representar, ou dar maior significado ao que se quer avaliar sendo válido, portanto, num contexto específico ${ }^{(6)}$.

Dessa forma, indicadores de avaliação da qualidade como formas numéricas ou não, são obtidos a partir dos
Assim, a especificidade da assistência de enfermagem em unidades neonatais exige profissionais bem preparados para a identificação rápida dos problemas e para a realização das intervenções necessárias. Nesse sentido, um dos aspectos que deve ser considerado nessa assistência é a manutenção da integridade da pele do RN.

A pele é um órgão capacitado à execução de múltiplas funções, sendo a principal delas a função de barreira de proteção para as estruturas internas do organismo, à ação de agentes externos, impedindo a perda de água e outras substâncias do meio interno, além de representar obstáculo à ação de radiações ultra-violetas pela ação da melanina. As outras funções da pele compreendem: proteção imunológica pelos componentes da derme; termorregulação pela sudorese, constrição e dilatação dos vasos cutâneos; percepção através da rede nervosa, e secreção sebácea, tendo importância também, por evitar as perdas de água e possuir propriedades antimicrobianas, além de conter os precursores da vitamina $\mathrm{D}^{(9)}$. 
Diante das especificidades anatômicas e fisiológicas da pele do neonato, que o suscetibilizam ao desenvolvimento de lesões de pele, manter a sua integridade é, portanto, algo especial, uma vez que preserva as funções de proteção e diminui os riscos de infecções decorrentes da hospitalização.

Dessa maneira, a manutenção da integridade da pele em pacientes hospitalizados é um importante aspecto da assistência à saúde que contribui com resultados positivos para o paciente. Por isso, desde 1994, a American Nurses Association passou a considerar como indicador, a integridade da pele para assegurar a qualidade da assistência de enfermagem ${ }^{(10)}$.

O cuidado com a pele do RN representa um fator de preocupação para os membros da equipe de enfermagem, já que a pele íntegra constitui uma barreira contra agentes do meio externo e protege as estruturas internas do organismo, além de ser um sinalizador para os problemas decorrentes da hospitalização como infecções e outros distúrbios do metabolismo.

Assim, o enfermeiro precisa conhecer as características do sistema tegumentar do neonato, avaliando-o criteriosamente, identificando os riscos inerentes às particularidades do RN e os riscos associados à terapêutica, direcionando a assistência de enfermagem prestada.

Considerando-se a constante necessidade de desenvolvimento profissional para atingir a excelência da qualidade da assistência3 prestada, entende-se que ao elucidar os fatores associados à manutenção da integridade da pele do RN por meio da aplicação de indicadores, estarão sendo fornecidos subsídios para a elaboração e efetivação de programas de capacitação.

Assim, o objetivo deste estudo foi o de propor um programa de capacitação para o pessoal de enfermagem, tendo por base um indicador de qualidade da assistência de enfermagem relacionado à manutenção da integridade da pele do $\mathrm{RN}$, durante um determinado período de internação hospitalar.

\section{MÉTODO}

O estudo de abordagem quantitativa, do tipo exploratório-descritivo, baseado no levantamento prospectivo de dados, foi desenvolvido na Unidade Neonatal do Hospital Universitário da Universidade de São Paulo (HU-USP).

Como proposta assistencial, o Departamento de Enfermagem do HU-USP adota o Sistema de Assistência de Enfermagem (SAE), iniciado em 1981, segundo os modelos teóricos de Horta (Teoria das Necessidades Humanas Básicas de 1979) e os conceitos de Orem (Teoria do Déficit do Autocuidado de 1985), que visam à assistência humanizada e individualizada, científica e de qualidade ${ }^{(11)}$.

Para isso, a Divisão de Enfermagem Materno-infantil do HU-USP, juntamente com as unidades relacionadas, estabeleceu padrões e critérios assistenciais objetivando assegurar a qualidade da assistência prestada ${ }^{(12)}$. Esses padrões, atualmente, vem sofrendo modificações com a implementação do Diagnóstico de Enfermagem, na tentativa de se adequarem melhor às necessidades da população atendida e elevar a qualidade da assistência de enfermagem prestada.

Na Unidade Neonatal do HU-USP, os cuidados com a pele do RN são realizados de acordo com padrões e critérios estabelecidos. Os principais cuidados são relacionados a procedimentos específicos como banho, curativo do coto umbilical, higiene perineal, coletas de exames e monitorização dos sinais vitais. Além disso, esses cuidados podem também estar relacionados à terapêutica instituída como cuidados com a fototerapia, administração de medicamentos, entre outros.

Dessa forma, ao admitir o RN na Unidade Neonatal, o enfermeiro inicia a assistência pela avaliação cuidadosa das condições do RN. Após essa avaliação, elabora propostas de cuidados de enfermagem, segundo o SAE adotado por esta instituição. Durante o período de internação, diariamente, após exame físico e observação rigorosa das condições do RN, são revistos o Diagnóstico, a Prescrição e a Evolução de Enfermagem e a assistência prestada pela equipe.

Assim, para a identificação das lesões de pele adquiridas durante a internação hospitalar, a população deste estudo foi constituída por 121 (100\%) RN admitidos na Unidade Neonatal no período de janeiro a maio de 2006 e que atenderam aos seguintes critérios de inclusão:

- Ter permanecido no mínimo 24 horas internado;

- Ter apresentado pele íntegra no momento da admissão; Ter no máximo 28 dias de vida no momento da admissão;

- Não apresentar malformações graves que afetassem a integridade da pele.

A coleta de dados foi realizada após a aprovação do projeto de pesquisa pelo Departamento de Enfermagem e Comitê de Ética em Pesquisa do HU-USP de acordo com a Resolução № 196/96 do Conselho Nacional de Saúde (Registro CEP: 623/05).

Para a coleta de dados foi elaborado um instrumento, visando à caracterização da população e a identificação das lesões de pele adquiridas, contendo duas partes.

$\mathrm{Na}$ Parte I, foram levantados os dados biológicos do recém-nascido como: registro do RN, data e hora do nascimento, sexo, classificação do peso e idade gestacional, procedência, total de dias de internação e presença de lesão de pele adquirida durante a internação.

Na Parte II, foram coletados dados relacionados às lesões de pele adquiridas durante a internação e aos fatores de risco associados, como: tipo de lesão de pele, dia de vida do RN em que a lesão apareceu, localização da lesão, presença ou não de dados relacionados às lesões de pele do neonato no Impresso de Diagnóstico/Evolução/Prescrição de Enfermagem, 
fatores de risco associados à lesão desenvolvida e relacionados à terapêutica utilizada, ao uso de adesivos, de medicamentos intravenosos e do sensor de oxímetro de pulso.

A coleta de dados foi efetuada pela pesquisadora na Unidade Neonatal diariamente, no período de janeiro a maio de 2006, iniciando pelo critério de inclusão do RN no estudo, no momento da admissão. Após esta etapa, foi preenchida a Parte I do instrumento, com dados coletados dos impressos Ficha do Recém-Nascido e Anotação de Enfermagem.

A Parte ll do instrumento foi preenchida diariamente, após a realização do exame físico detalhado do $\mathrm{RN}$ e a verificação dos impressos de Prescrição Médica, Anotação de Enfermagem, Diagnóstico/Evolução/Prescrição de Enfermagem.

Os resultados encontrados foram armazenados na Planilha Eletrônica Excel 2003, para posterior processamento e para serem apresentados na forma de tabelas e gráficos, em números absolutos e índices percentuais. As tabelas foram editadas no programa Word 2003 e submetidas a análise estatística.
Esses resultados fundamentaram a elaboração de uma proposta de capacitação para o pessoal de enfermagem da unidade estudada.

\section{RESULTADOS E DISCUSSÃO}

Para a caracterização da população estudada, foram identificados dados referentes ao sexo, classificação do peso e idade gestacional, procedência, dias de internação, diagnóstico médico de admissão e presença de lesão de pele adquirida durante a internação.

Dos 121 (100\%) RN estudados, 73 (60,3\%) eram do sexo masculino e $48(39,7 \%)$ do sexo feminino.

Na Tabela 1 estão apresentados os dados referentes à classificação dos neonatos de acordo com as variáveis peso e idade gestacional. Essa classificação fornece orientação para o controle do neonato e prediz os riscos de mortalidade, sendo considerados como pré-requisitos para a definição de normalidade.

Tabela 1 - Distribuição dos recém-nascidos na Unidade Neonatal segundo a classificação do peso e idade gestacional, HU-USP - São Paulo - 2006

\begin{tabular}{lcc}
\hline Classificação do peso e idade gestacional & N & \% \\
\hline RNT-AIG- RN termo-adequado para a idade gestacional & 83 & 68,6 \\
RNT-GIG RN termo-grande para a idade gestacional & 13 & 10,8 \\
RNT-PIG RN termo-pequeno para a idade gestacional & 8 & 6,6 \\
RNPT-AIG RN pré-termo-adequado para a idade gestacional & 16 & 13,2 \\
RNPT-GIG RN pré-termo-grande para a idade gestacional & 1 & 0,8 \\
\hline Total & $\mathbf{1 2 1}$ & $\mathbf{1 0 0 , 0}$ \\
\hline
\end{tabular}

A Tabela 1 mostra que a maioria dos RN $83(68,6 \%)$ foi classificada como RNT-AIG; 13 (10,8\%) como RNT-GIG e $8(6,6 \%)$ como RNT-PIG, constituindo assim, uma população de baixo risco de desenvolvimento de problemas relacionados a maturidade física.

Entre os pré-termos, $16(13,2 \%)$ foram classificados como RNPT-AIG e apenas $1(0,8 \%)$ como RNPT-GIG.

Tabela 2 - Distribuição dos recém-nascidos na Unidade Neonatal segundo a procedência, HU-USP - São Paulo - 2006

\begin{tabular}{lcc}
\hline Procedência & $\mathbf{N}$ & $\mathbf{\%}$ \\
\hline Centro Obstétrico & 67 & 55,4 \\
Alojamento Conjunto & 31 & 25,6 \\
Pronto-Socorro Infantil & 13 & 10,8 \\
Ambulatório & 5 & 4,1 \\
Unidade de Terapia Intensiva & 5 & 4,1 \\
\hline Total & $\mathbf{1 2 1}$ & $\mathbf{1 0 0 , 0}$ \\
\hline
\end{tabular}

Conforme mostra a Tabela 2, a maioria dos neonatos estudados, 67 (55,4\%) foram procedentes do Centro Obstétrico, provavelmente por terem apresentado problemas de adaptação a vida extra uterina.

Os principais diagnósticos médicos de internação foram: Desconforto Respiratório Precoce, Risco Infeccioso e Icterícia Neonatal.

Os 121 RN estudados somaram um total de 732 pacientes/dia, dentre eles, 94 (77\%) neonatos desenvolveram algum tipo de lesões durante sua internação, somando um total de 230 lesões de pele.

Dessa forma, a taxa de incidência desses eventos foi de 0,314 lesão por paciente-dia e média de 1,9 lesão/ RN, caracterizando uma população com um risco elevado de desenvolvimento de problemas de pele durante o período de internação hospitalar.

A Tabela 3 apresenta os tipos de lesões de pele adquiridas pelos neonatos durante o período de internação hospitalar. 
Tabela 3 - Distribuição dos tipos de lesões de pele adquiridas pelos RN durante a internação na Unidade Neonatal, HU-USP - São Paulo - 2006

\begin{tabular}{lcc}
\hline Tipo de lesão & $\mathbf{N}$ & $\mathbf{\%}$ \\
\hline Equimose & 117 & 50,9 \\
Eritema perineal & 66 & 28,7 \\
Monilíase & 19 & 8,3 \\
Infiltração & 8 & 3,5 \\
Hematoma & 7 & 3,0 \\
Erosão & 5 & 2,2 \\
Fissura & 4 & 1,7 \\
Escoriação & 2 & 0,9 \\
Abcesso & 1 & 0,4 \\
Lesões bolhosas - impetigo & 1 & 0,4 \\
\hline Total & $\mathbf{2 3 0}$ & $\mathbf{1 0 0 , 0}$ \\
\hline
\end{tabular}

Das 230 lesões adquiridas durante a internação, 117 $(50,9 \%)$ foram equimoses, $66(28,6 \%)$ foram eritemas perineais e $19(8,3 \%)$ monilíases, sendo que o mesmo RN poderia ter desenvolvido mais de uma lesão.

Cabe ressaltar que após o nascimento, fatores significantes do meio ambiente e comportamental podem afetar a estrutura e função da pele. Esses fatores incluem mudanças na temperatura do ambiente, alterações no ressecamento e umidade da superfície da pele e o contato intermitente com a fricção das roupas, cobertores e fraldas ${ }^{(13)}$.

Assim, lesões como erosões e fissuras poderiam ter sido causadas pelo ressecamento fisiológico da pele do neonato e ocorreram em menor número.

Dessa maneira, a internação hospitalar expõe o neonato a outros fatores relacionados ao meio ambiente, aos cuidados prestados, à investigação diagnóstica e à terapêutica medicamentosa.

A maioria das lesões surgiu nos primeiros três dias após a internação hospitalar, sugerindo a necessidade de estabelecimento de intervenções rápidas para a prevenção de suas ocorrências. Na Figura 1, foram comparados os dias de internação entre os neonatos que não tiveram lesões de pele e aqueles que adquiriram alguma lesão durante a internação.

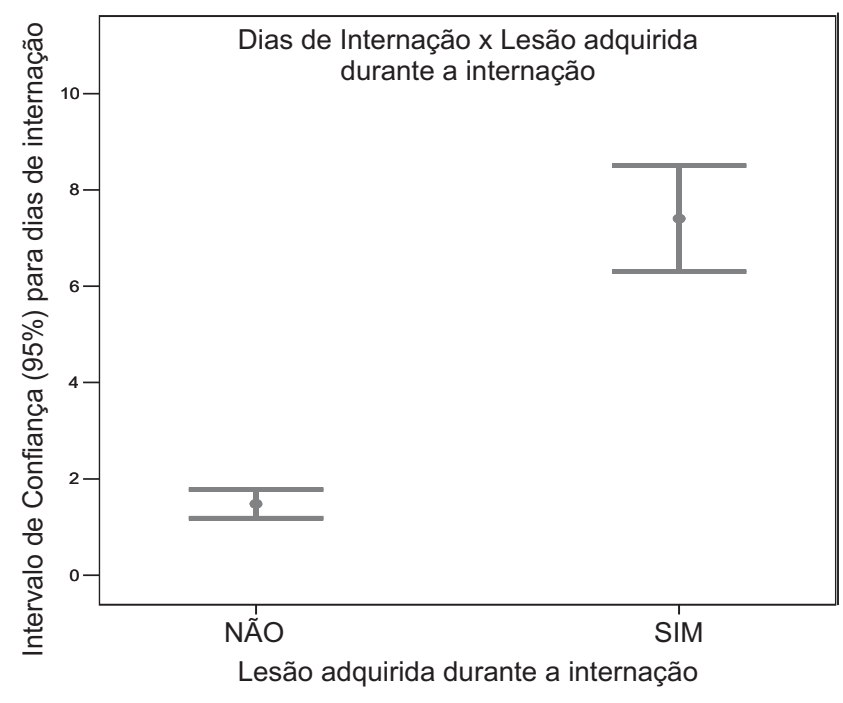

Figura 1 - Distribuição do número de dias de internação hospitalar e a presença de lesão adquirida por RN na Unidade Neonatal, HUUSP - São Paulo - 2006

A Figura 1 demonstra que os RN que desenvolveram os tipos de lesões de pele mais freqüentes identificadas apresentaram em média, maior tempo de internação comparado com os que não as desenvolveram.

O surgimento da primeira lesão ocorreu, em sua maioria nos membros superiores, e foram associadas a lesões do tipo equimose, hematomas e infiltrações, possivelmente por serem os primeiros locais de escolha para coleta de exames de sangue e introdução de dispositivos para terapêutica intravenosa;

Ao se comparar a presença de lesão com o registro desses dados em impressos do SAE, verificou-se que foram levantadas, pelo enfermeiro, lesões do tipo equimoses, eritema perineal monilíases;

Lesões do tipo escoriação, erosão, fissuras e hematomas não foram registradas no SAE no momento da coleta dos dados, sendo identificadas pela pesquisadora no exame físico, sugerindo uma mecanização desse processo e/ ou a necessidade de capacitação desses profissionais para a identificação precoce dessas lesões. 
Tabela 4 - Distribuição dos tipos de lesões de pele adquiridas pelos RN durante a internação na Unidade Neonatal segundo os fatores de risco associados, HU-USP - São Paulo - 2006

\begin{tabular}{|c|c|c|c|c|c|c|c|c|c|c|}
\hline \multirow{4}{*}{ Tipos de lesão } & \multicolumn{10}{|c|}{ Fator de Risco } \\
\hline & \multicolumn{3}{|c|}{ Adesivo } & & \multicolumn{3}{|c|}{ Terapia IV } & \multirow{2}{*}{\multicolumn{3}{|c|}{$\begin{array}{c}\text { Oxímetro } \\
\text { NÃO }\end{array}$}} \\
\hline & SIM & & NÃO & & SIM & & NÃO & & & \\
\hline & $\mathrm{N}$ & $\%$ & $\mathrm{~N}$ & $\%$ & $\mathrm{~N}$ & $\%$ & $\mathrm{~N}$ & $\%$ & $\mathrm{~N}$ & $\%$ \\
\hline Equimose & & & 117 & $100,0 \%$ & 99 & $84,6 \%$ & 18 & $15,4 \%$ & 117 & $100,0 \%$ \\
\hline Eritema perineal & & & 66 & $100,0 \%$ & 51 & $77,3 \%$ & 15 & $22,7 \%$ & 66 & $100,0 \%$ \\
\hline Monilíase & & & 19 & $100,0 \%$ & 18 & $94,7 \%$ & 1 & $5,3 \%$ & 19 & $100,0 \%$ \\
\hline Infilttração & & & 8 & $100,0 \%$ & 8 & $100,0 \%$ & & & 8 & $100,0 \%$ \\
\hline Hematoma & & & 7 & $100,0 \%$ & 6 & $85,7 \%$ & & & 7 & $100,0 \%$ \\
\hline Fissura & & & 4 & $100,0 \%$ & & & 4 & $100,0 \%$ & 4 & $100,0 \%$ \\
\hline Erosão & 3 & $60,0 \%$ & 2 & $40,0 \%$ & 1 & $20,0 \%$ & 4 & $80,0 \%$ & 5 & $100,0 \%$ \\
\hline Escoriação & 1 & $50,0 \%$ & 1 & $50,0 \%$ & 2 & $100,0 \%$ & & & 2 & $100,0 \%$ \\
\hline Abcesso & & & 1 & $100,0 \%$ & & & 1 & $100,0 \%$ & 1 & $100,0 \%$ \\
\hline $\begin{array}{l}\text { Lesões bolhosas } \\
\text { impetigo }\end{array}$ & & & 1 & $100 \%$ & & & 1 & $100,0 \%$ & 1 & $100,0 \%$ \\
\hline
\end{tabular}

De acordo com a Tabela 4, quanto aos fatores de risco foram considerados:

- o uso de adesivos associado a lesões do tipo erosão e escoriação, provavelmente pela dificuldade na sua remoção;

- a terapêutica intravenosa associada com a maioria das lesões identificadas, como lesões do tipo equimoses, infiltrações,hematomas, erosão, infiltração e erupções cutâneas na região abrangida pela fralda;

- o uso do sensor do oxímetro não apresentou relação com as lesões encontradas.

Dessa forma, os achados desse estudo sugerem que a manutenção da integridade da pele do RN durante a internação hospitalar pode prevenir a ocorrência de efeitos adversos das terapêuticas instituídas, reduzindo os riscos de ocorrências iatrogênicas decorrentes da assistência prestada.

As variáveis estudadas constituem dados concretos que referendam a necessidade de se propor um programa de capacitação para o pessoal de enfermagem com a finalidade de capacitar os enfermeiros, técnicos e auxiliares de enfermagem para o alcance da excelência da qualidade do serviço de enfermagem.

Para isso, a base teórica desse Programa está apoiada nos princípios da elaboração de um planejamento coletivo e no desenvolvimento de competências técnico-científicas, sócio-educativas e ético-políticas da equipe de enfermagem.

Para essa elaboração foi definida como estratégia participativa, a formação de agentes multiplicadores que, por sua vez, replicam a experiência vivida através de oficinas de trabalho, junto aos membros da equipe de enfermagem que também prestam assistência nessa unidade.

Um estudo realizado em unidades de internação de um hospital privado de São Paulo apresentou um programa de capacitação para enfermeiros recém-admitidos com base desenvolvimento de competências, destacando resultados positivos após sua implementação, pois constitui uma maneira construtiva de se perceber o outro e de se desenvolver a cooperação e a solidariedade na equipe de enfermagem $^{(14)}$.

Assim, visando facilitar o processo de implantação do Programa, didaticamente, foram considerados, na sua elaboração, cinco momentos: Primeiro- Diagnóstico Situacional, Segundo- Definição dos Objetivos segundo as competências, Terceiro- Elaboração do Programa e Definição das Estratégias, Quarto- Implementação e Quinto- Avaliação.

Para o diagnóstico situacional, o resultado da caracterização das lesões de pele adquiridas pelos RN durante o período de internação na unidade neonatal, constituiu a etapa inicial, sendo necessária uma ampla discussão, desses achados, com os membros da equipe de enfermagem, de forma participativa, estimulando a reflexão crítica da realidade.

Cabe ressaltar que a pesquisa tem sido apontada como um instrumento importante no auxilio, na validação de práticas já consagradas e na transformação crítica do cotidiano pela possibilidade de direcionar a realização das mudanças necessárias ${ }^{(15)}$.

Neste sentido, segundo pressupostos da gerência participativa, os profissionais envolvidos deverão ser estimulados a participar da busca de soluções alternativas, contribuindo, pelo compartilhamento de experiências vividas, para o crescimento pessoal, profissional e coletivo, possibilitando a transformação da realidade.

Após essa etapa, torna-se possível a identificação das reais necessidades de capacitação da equipe relacionada aos resultados desse indicador de qualidade e assim, viabilizar a definição dos objetivos e metas a serem alcançados, constituindo o Segundo momento. 
De forma preliminar, o programa teve como objetivo geral desenvolver no pessoal de enfermagem competências técnico-científicas, ético-políticas e sócio-educativas visando à capacitação da equipe e a melhoria da qualidade da assistência de enfermagem prestada na Unidade Neonatal.

Diante disso, os objetivos específicos foram:

De acordo com as Competências técnico-científicas

- Conhecer a Anatomia e a Fisiologia da pele do RN de termo e do prematuro;

- Identificar os fatores de risco para lesões de pele;

- Identificar precocemente as lesões de pele;

- Conhecer as intervenções necessárias diante do risco e/ou das lesões identificadas;

- Conhecer as formas de avaliação de qualidade da assistência prestada.

De acordo com as Competências sócio-educativas

- Sensibilizar a equipe de enfermagem quanto à importância da manutenção da integridade da pele;

- Rever os padrões e critérios estabelecidos para a assistência de enfermagem;

- Promover situações de reflexão que estimulem a equipe a melhorar a qualidade da assistência prestada;

\section{De acordo com as Competências ético-políticas}

- Desenvolver a capacidade de analisar criticamente os cuidados prestados;

- Reorganizar os padrões, critérios e condições institucionais estabelecidos para a manutenção da integridade da pele do $\mathrm{RN}$;

- Elaborar políticas educacionais para a capacitação de pessoal voltada para as necessidades pesquisadas nessa unidade.

Para o momento de Elaboração do programa e definição das estratégias de trabalho, cabe salientar que essas estratégias devem promover a participação efetiva dos membros da equipe, permitindo a reflexão crítica da realidade, para promover sua transformação, acreditando que a mesma se constrói na interação com o ambiente ${ }^{(16)}$.

Nessa direção, o processo de desenvolvimento de recursos humanos tem sido facilitado pela ação de agentes multiplicadores como estratégia de trabalho. Para isso, profissionais de uma determinada área são responsáveis pelo treinamento e desenvolvimento da equipe dessa área. $\mathrm{O}$ agente multiplicador pertence ao grupo, o que favorece a troca de informações, estimulando a motivação e a cooperação entre os membros e facilitando todo o processo ${ }^{(17-18)}$.

Dessa forma, poderá ser selecionado um enfermeiro em cada turno de trabalho que atuará como agente multipli- cador da equipe de enfermagem, coordenando um grupo de enfermeiros interessados no desenvolvimento desse tema. Esses profissionais serão capacitados para a execução do programa e ficarão responsáveis pelo treinamento e desenvolvimento dos técnicos e auxiliares de enfermagem do seu turno de trabalho.

Assim, para atuação dos agentes multiplicadores poderão ser desenvolvidas oficinas de trabalho e o acompanhamento do funcionário durante sua jornada de trabalho, visando à identificação de problemas relacionados ao tema proposto, trazendo assim, benefícios para a implementação da proposta.

Nas oficinas de trabalho, poderão ser incluídos cursos, palestras, seminários e jornadas desenvolvidas na instituição para o aprofundamento teórico do tema, sendo realizadas na própria Unidade Neonatal, dividindo em grupos de quatro ou cinco pessoas facilitando, dessa forma, a interação e o compartilhamento das experiências vividas.

No Quarto momento, a implantação do programa ocorrerá após a sua aprovação pelos pares e pelas instâncias superiores da instituição. Essa fase pode contribuir para o desenvolvimento das competências relacionadas à manutenção da integridade da pele do neonato, utilizando estratégias participativas que favoreçam o pensamento crítico sobre a realidade vivida.

Finalmente, no momento da avaliação, será verificada a pertinência dos conteúdos propostos, a adequação das estratégias e dos recursos de ensino adotados, devendo incluir também a avaliação dos resultados, verificando-se o custo-benefício do investimento ${ }^{(18)}$.

Para tanto, há necessidade de registros e controles eficientes e contínuos que servirão como parâmetros para a aferição dos custos e para a avaliação do desempenho futuro dos participantes do programa com a finalidade de mostrar o impacto, desse treinamento, e conseqüentemente, o retorno de futuros investimentos ${ }^{(19)}$.

A quantificação das variáveis relacionadas ao indicador de qualidade integridade da pele do neonato, poderá servir como parâmetro para a avaliação do Programa de Capacitação estabelecendo assim, a mensuração de possíveis mudanças nos padrões de qualidade da assistência de enfermagem prestada e construindo séries históricas para o monitoramento desse indicador.

Dessa forma, deverá ser elaborado um instrumento que possibilite a avaliação dos investimentos obtidos e dos resultados alcançados, através do registro sistemático dos dados.

\section{CONCLUSÕES}

A realização deste estudo possibilitou a identificação das reais necessidades de capacitação da equipe de enfermagem relacionadas à manutenção da integridade da pele do neonato durante sua internação na Unidade Neonatal, 
possibilitando assim, a elaboração de um programa de capacitação para o pessoal de enfermagem.

Identificou-se uma taxa de incidência de 0,314 lesão por paciente-dia com média de 1,9 lesão/ RN, caracterizando uma população com um risco elevado de desenvolvimento de problemas de pele durante o período de internação hospitalar, necessitando assim, de maiores investimentos no preparo dos profissionais de enfermagem visando de minimizar a ocorrência desses eventos.

Acredita-se que o investimento na capacitação da equipe de enfermagem segundo as competências técnico-cien-

\section{REFERÊNCIAS}

1. Leitão RER, Kurcgant P. Qualidade na prática gerencial da enfermagem: as duas faces da mesma moeda. Niterói: Intertexto; 2004.

2. Nogueira RP. Perspectivas da qualidade em saúde. Rio de Janeiro: Qualitymark; 1994.

3. Donabedian A. The role of outcomes in quality assesment and assurance. Qual Rev Bull. 1992;20(6):975-92.

4. Adami NP. A melhora da qualidade nos serviços de enfermagem. Acta Paul Enferm. 2000;13(n.esp):190-6.

5. Bittar OJNV. Indicadores de qualidade e quantidade em saúde. Rev Adm Saúde. 2001;12(3):21-8.

6. Tanaka OY, Melo C. Avaliação de programas de saúde do adolescente: um modo de fazer. São Paulo: EDUSP; 2001.

7. Teixeira JDR, Camargo FA, Tronchin DMR, Melleiro MM. A elaboração de indicadores de qualidade da assistência de enfermagem nos períodos puerperal e neonatal. Rev Enferm UERJ. 2006;14(2):271-8.

8. Soboll MLMS, Carvalho AO, Eduardo MBP, Tanaka OY. Sistemas de informação em saúde, mecanismo de controle, de auditoria e de avaliação. In: Westphal MF, Almeida ES, organizadores. Gestão de serviços de saúde-descentralização, municipalização do SUS. São Paulo: EDUSP, 2001. p. 205-54.

9. Sampaio SAP, Rivitti EA. Dermatologia. 2ª ed. São Paulo: Artes Médicas; 2000.

10. Meraviglia M, Becker $H$, Grobe SJ, King M. Maintence of skin integrity as a clinical indicator of nursing care. Adv Skin Wound Care. 2002;15(1):24-29.

11. Gualda DMR. Organização do Departamento de Enfermagem do Hospital Universitário da Universidade de São Paulo: filosofia e pressupostos acadêmicos-assistenciais. In: Cianciarullo TI, Gualda DMR, Melleiro MM, Anabuki MH, organizadoras. Sistema de assistência de enfermagem: evolução e tendências. 3aed. São Paulo: Ícone; 2005. p. 93-108. tíficas, sócio-educativas e ético-políticas, pode contribuir para a diminuição do índice desse indicador de qualidade, garantindo o aperfeiçoamento dos padrões e critérios de assistência ao RN na Unidade Neonatal do HU/USP e beneficiar tanto o paciente como a equipe de enfermagem.

Salienta-se ainda que, futuramente, a realização de outros estudos pode validar a efetividade da proposta elabora$\mathrm{da}$, comparando os resultados desse indicador de qualidade pela construção de séries históricas, além de quantificar e identificar diferentes necessidades de capacitação.

12. Florentino LC, Soares AVN, Souza TM, Spir EG, Andreoni S, Bálsamo AC. Elaborando e desenvolvendo padrões e critérios de qualidade na assistência perinatal. In: Cianciarullo $\mathrm{TI}$, Gualda DMR, Melleiro MM. C\&Q Indicadores de qualidade: uma abordagem perinatal. São Paulo: Ícone; 1998. p. 77-137.

13. Visscher MO, Chatterjee R, Munson KA, Bare DE, Hoath SB. Development of diaper rash in the newborn. Pediatr Dermatol. 2000;17(1):52-7.

14. Siqueira ILCP, Kurcgant P. Estratégia de capacitação de enfermeiros recém-admitidos em unidades de internação geral. Rev Esc Enferm USP. 2005;39(3):251-7.

15. Castilho V. Educação continuada em enfermagem: a pesquisa como possibilidade de desenvolvimento de pessoal. Mundo Saúde. 2000;24(5):357-60.

16. Salum NC, Prado ML. Educação continuada no trabalho: uma perspectiva de transformação da prática e valorização do trabalhador(a) de enfermagem. Texto Contexto Enferm. 2000;9(2):298-311.

17. Bicudo AMC, Silva MCM, Cunha ICKO. Percepção das enfermeiras que atuam como agentes multiplicadores no treinamento da equipe de enfermagem. Acta Paul Enferm. 2004;17(3):305-10.

18. Peres HHC, Leite MMJ, Gonçalves VLM. Educação continuada: recrutamento e seleção, treinamento e desenvolvimento e avaliação de desempenho profissional. In: Kurcgant $P$, coordenadora. Gerenciamento em enfermagem. São Paulo: Guanabara Koogan; 2005. p. 139-49.

19. Jericó MC, Castilho V. O investimento financeiro em recursos humanos: uma experiência em hospital. Rev Lat Am Enferm. 2004;12(5):707-12. 